\title{
The effect of trichostatin-A and tumor necrosis factor on expression of splice variants of the MaxiK and L-type channels in human myometrium
}

\author{
Sarah L. Waite ${ }^{1}$, Saurabh V. Gandhi ${ }^{2}$, Raheela N. Khan ${ }^{3}$ and Neil R. Chapman ${ }^{1 *}$ \\ ${ }^{1}$ Academic Unit of Reproductive and Developmental Medicine, Department of Human Metabolism, University of Sheffield, Sheffield, UK \\ ${ }^{2}$ Department of Obstetrics and Gynaecology, Sheffield Teaching Hospitals NHS Foundation Trust, Sheffield, UK \\ ${ }^{3}$ Division of Medical Sciences and Graduate Entry Medicine, School of Medicine, Royal Derby Hospital, University of Nottingham, Derby, UK
}

Edited by:

Gaetano Santulli, Columbia, USA

Reviewed by:

Bin-Nan Wu, Kaohsiung Medical University, Taiwan

Richard David Veenstra, SUNY

Upstate Medical University, USA

\section{*Correspondence:}

Neil R. Chapman, Academic Unit of

Reproductive and Developmental

Medicine, Department of Human

Metabolism, University of Sheffield,

Level 4, Jessop Wing, Tree Root

Walk, Sheffield S10 2SF, South

Yorkshire, UK

e-mail:n.r.chapman@sheffield.ac.uk
The onset of human parturition is associated with up-regulation of pro-inflammatory cytokines including tumor necrosis factor (TNF) as well as changes in ion flux, principally $\mathrm{Ca}^{2+}$ and $\mathrm{K}^{+}$, across the myometrial myocytes membrane. Elevation of intra-cellular $\mathrm{Ca}^{2+}$ from the sarcoplasmic reticulum opens L-type $\mathrm{Ca}^{2+}$ channels (LTCCs); in turn this increased calcium level activates MaxiK channels leading to relaxation. While the nature of how this cross-talk is governed remains unclear, our previous work demonstrated that the pro-inflammatory cytokine, TNF, and the histone deacetylase inhibitor, Trichostatin-A (TSA), exerted opposing effects on the expression of the pro-quiescent Gas gene in human myometrial cells. Consequently, in this study we demonstrate that the different channel splice variants for both MaxiK and LTCC are expressed in primary myometrial myocytes. MaxiK mRNA expression was sensitive to TSA stimulation, this causing repression of the $M 1, M 3$, and M4 splice variants. A small but not statistically significantly increase in MaxiK expression was also seen in response to TNF. In contrast to this, expression of LTCC splice variants was seen to be influenced by both TNF and TSA. TNF induced overall increase in total LTCC expression while TSA stimulated a dual effect: causing induction of LTCC exon 8 expression but repressing expression of other LTCC splice variants including that encoding exons 30,31,33, and 34, exons 30-34 and exons 40-43. The significance of these observations is discussed herein.

Keywords: parturition, MaxiK, LTCC, splice variants, TNF, TSA

\section{INTRODUCTION}

In the developed world, premature birth (that before 37 weeks completed gestation) complicates $6-12 \%$ of pregnancies (Khashan et al., 2010). Annually it is estimated that 1.1 million babies worldwide die from being born prematurely (Blencowe et al., 2012; Chang et al., 2013), surviving infants having an elevated risk of major long-term mental and physical handicap (Marlow et al., 2005; Costeloe et al., 2012). Moreover, such infants also have a disproportionate effect on health-care budgets worldwide: a recent UK estimate of the total cost of preterm birth to the public sector was $£ 2.95$ billion (Mangham et al., 2009). Tocolytic therapies (drugs which stop premature contractions of the womb) are few in number and are associated with complications for both infant and mother (Oei, 2006). Since the antenatal health of a baby is a major predictor of adult morbidity (Nijland et al., 2008), reducing the incidence of premature birth is an unequivocal healthcare goal. Indeed, a recent review suggested a conservative target of relative reduction of the pre-term birth rate by $5 \%$ by 2015 (Chang et al., 2013). Until the fundamental biology of the pregnant human uterus is defined, however, that target is unlikely to be realized.

The smooth muscle of the non-pregnant uterus, the myometrium, naturally contracts when subjected to stretch.
During normal gestation, however, the pregnant myometrium must stretch to accommodate the growing baby. As such, the myometrium enters a prolonged phase of relaxation which is termed quiescence. This quiescent state is important because it facilitates normal growth and development of the baby. The quiescent state is characterized by slow wave potentials where the membrane potential cycles between depolarization and repolarizations without reaching the threshold level to produce an action potential (Parkington et al., 1999; Smith et al., 2002; Aguilar and Mitchell, 2010). At term, this quiescent state ends and these slow wave potentials become frequent synchronized action potentials during which the membrane potential rapidly rises and falls (Wilde and Marshall, 1988) - -i.e., the myometrium undergoes the rhythmic contractions seen in labor to ensure the baby is delivered (Khan et al., 1993b, 1997, 1998a,b; Tribe et al., 2000; Shmygol et al., 2007). Unfortunately, the fundamental nature of the molecular mechanisms regulating human quiescence and labor are poorly understood, the corollary being that this greatly limits our understanding of how parturition begins too early leading to premature birth of the baby.

In terms of myometrial quiescence, this period of pro-longed relaxation occurs when calcium is maintained below the threshold for depolarization that would trigger calcium entry. One 
mechanism by which this is achieved involves potassium channels that either repolarize the cell membrane thus causing the calcium channels to close and therefore prevent further calcium from entering the cell (Khan et al., 1993a,b, 1997; Shmygol et al., 2007) or maintaining the cell resting membrane potential through potassium leak channels of the two-pore domain potassium (K2P) channel family (Buxton et al., 2010) and others (Knock and Aaronson, 1999; Tribe et al., 2000). Calcium activated potassium channels, including MaxiK, have an important role regulating action potentials in some smooth muscles. Calcium influx through L-type $\mathrm{Ca}^{2+}$ channels (LTCCs) is the principle source driving smooth muscle contractility and while many types of potassium channels counteract the influx of calcium and maintain or promote smooth muscle relaxation, the large-conductance calcium-activated potassium channel (BKCa, MaxiK) is activated by intracellular calcium and acts to oppose the depolarization (Toro et al., 1990; Khan et al., 1993b, 1997) and so responds to both changes in calcium concentration and membrane potential. Calcium influx through LTCCs is the principle source driving smooth muscle contractility and therefore calcium activated potassium channels, including MaxiK, have an important role regulating action potentials in some smooth muscles. Thus, fine tuning of the activity of both MaxiK and LTCC would appear to be, in part, a key process for maintenance of quiescence during gestation and contraction during labor (Khan et al., 1993b, 1997, 1998a,b; Brainard et al., 2005; Shmygol et al., 2007).

The MaxiK is thought to be one of the key potassium channels within the myometrium (Anwer et al., 1993; Khan et al., 1993b, 1997, 1998a,b; Perez et al., 1993; Tribe et al., 2000; Shmygol et al., 2007). The MaxiK channel itself is a tetrameric structure containing a pore-forming $\alpha$-subunit and a regulatory $\beta$-subunit (Wallner et al., 1995; Lu et al., 2006) and is documented to have a number of splice variants (Song et al., 1999; Curley et al., 2004). Briefly, these include the MK44 variant (M1) which can either encode a 132 bp insert between exons 1 and 2 or have this insert omitted. The channels containing the insert have diminished sensitivity to intracellular calcium and also voltage (Korovkina et al., 2001). The second variant involves the mutually exclusive use of either exons 10 and 11 or 11 and 12 (M2), which can cause a truncation of the channel protein (Curley et al., 2004). Three variants of exon 19 have also been described these are a $3^{\prime}$ truncation of the exon, skipping of the exon and the use of the entire of exon 19, (M3), the effect of these different variants has not been fully explored (Curley et al., 2004). The Stress Regulated Exon (STREX/M4) is another well described variant which involves the differential utilization of exons 22 and 23, channels utilizing both exons have been shown to have increased mechano-sensitivity and hypoxia inhibition (McCartney et al., 2005; Lu et al., 2006; Wang et al., 2010). Finally there is a variant involving the use or omission of exon 29, (M5), utilization of exon 29 creates channels with increased activation rates and modified calcium co-operativity (Ha et al., 2000; Yan et al., 2008).

It is clear that alternate splicing of the MaxiK $\alpha$-subunit can alter its sensitivity to calcium, voltage, protein phosphorylation and cellular localization: all are methods by which the diversity of $\mathrm{K}^{+}$channel signaling arises (Soloff et al., 2004; Torres et al.,
2007; Aguilar and Mitchell, 2010). This provides a mechanism for fine tuning the channels response to a diverse range of regulatory stimuli. Indeed the expression of different MaxiK channel isoforms is tissue and stimulus-specific (Lu et al., 2006) and may be a mechanism by which uterine contractility can be modulated during gestation.

The LTCC is the predominant calcium channel in the myometrium (Tezuka et al., 1995; Parkington et al., 1999; Collins et al., 2000). The LTCC is a type of voltage-dependent calcium channel consisting of five subunits $(\alpha 1, \alpha 2, \beta, \gamma$, and $\delta)$ with the $\alpha 1$-subunit defining most of the channel's properties (JurkatRott and Lehmann-Horn, 2004; Aguilar and Mitchell, 2010). LTCC are responsible for normal myocardial and vascular smooth muscle contractility and are also subject to splicing similar to MaxiK (Jurkat-Rott and Lehmann-Horn, 2004; Tang et al., 2004; Aguilar and Mitchell, 2010). Briefly, these include the mutually exclusive use of exons $1,1 \mathrm{~b}$, or 1c (L1), these affect membrane expression of the channel (Snutch et al., 1991; Soldatov, 1992; Bannister et al., 2011). Exons 8 and $8^{*}$ are also mutually exclusively expressed (L4), use of exon $8^{*}$ results in channels with lower Dihydropyridine (DHP) block sensitivity, more rapid activation and slower inactivation rates (Soldatov, 1992). Exons 30, 31, 32, 33 , and 34 (L10) can also be utilized in a number of different combinations (L10). These alter the size and rigidity of the linker between the S3 and S4 segments of the channel, with a shorter linker resulting in channels with slower gating kinetics and longer one resulting in channels with faster gating kinetics (Perez-Reyes et al., 1990; Yang et al., 2000). Finally, splice site 11/12 (L11/12) includes exons 40,41,42, and 43, variants in this region include the use of exon $40 \mathrm{~A}$ (exon 40 with a $19 \mathrm{bp}$ deletion), exon $40 \mathrm{~B}$ (exon 40 with a $125 \mathrm{bp}$ addition), exon $41 \mathrm{~A}$ and exon 43 with an additional $132 \mathrm{bp}$ (Gerhardstein et al., 2000). These variations affect calmodulin binding, calcium dependant inactivation and the presence or absence of a cAMP dependant protein kinase A site (Perez-Reyes et al., 1990; Snutch et al., 1991; Diebold et al., 1992; Soldatov, 1992, 1994; Tang et al., 2004).

Given the abundance of MaxiK in the myometrium and its links with $\mathrm{Ca}^{2+}$ flux via the LTCC, it is clear that these channel families play a pivotal role in governing myometrial quiescence and subsequent phenotypic change to uterine contractility. By responding to calcium influx the MaxiK channels provide an effective mechanism of negative feedback of to maintain relaxation (Khan et al., 1993b, 1997, 1998b, 2001). Moreover, we believe that the balance between LTCCs and MaxiK channels determines whether quiescence or contractility occurs within the myometrium.

In the human myometrium, the cessation of uterine quiescence and the onset of both normal and preterm labor are associated with a number of pro-inflammatory cytokines, including, but not limited to, IL-1 $\beta$, tumor necrosis factor (TNF), and IL-8, which are regulated by a family of transcription factors collectively referred to as Nuclear Factor kappaB (NFкB; reviewed in Cookson and Chapman, 2010). Our pilot promoter array studies demonstrate that the genomic regulatory regions of both MaxiK and the LTCC are bound by NFKB RelA in response to TNF (Cookson, 2010). Moreover, TNF was seen to induce nuclear localization of both channels in primary myometrial cells. 
We and others have previously demonstrated that external agents such as TNF (potent pro-inflammatory cytokine) can induce myometrial contractility while other compounds, namely trichostatin-A (TSA), can promote myometrial relaxation in isolated human smooth muscle strips (Lu et al., 1999; Moynihan et al., 2008; Webster et al., 2013). While the exact means by which this process occurred could not be elucidated, that study also demonstrated that TSA caused an up-regulation of the GTPbinding protein, Gas, while TNF had the reciprocal function and repressed the Gas promoter. The repression was due to recruitment of an enzyme, histone deacetylase-1 (HDAC-1) to the regulatory region of the Gas gene (Webster et al., 2013). Together, these data illustrated that while TSA can induce a relaxed myometrial phenotype required to stop muscle contraction, it could not overcome the uterine inflammation induced by those proinflammatory mediators, including TNF, known to be present in the uterus during labor.

In our view, our previous data suggests that there is a molecular conflict between TSA and TNF which we believe involves ion channel function possibly through the Gas subunit. Indeed, both MaxiK and LTCC are linked to adenylyl cyclase activity and $\beta 2$-adrenergic receptor function (Toro et al., 1990; Carney et al., 2002; Chanrachakul et al., 2004; Liu et al., 2004; Ledoux et al., 2006; Torres et al., 2007) which, when engaged, promote smooth muscle relaxation through increased Gas activity, subsequent elevations in intra-cellular cAMP and efflux of $\mathrm{K}^{+}$via MaxiK (Chanrachakul et al., 2004; Aguilar and Mitchell, 2010). It is likely, however, that both agents would also influence both ion channel expression and subsequent RNA splicing, which, in turn, would also modulate myometrial contractility. Given the effects we have previously reported for both TNF and TSA on primary human myometrium and myometrial myocytes (Webster et al., 2013), in this study we wished to examine the expression, localization, and splicing pattern of both the MaxiK channel and the LTCC in primary human myometrial cells and define whether these parameters were influenced by TNF and TSA.

\section{MATERIALS AND METHODS MYOMETRIAL BIOPSY COLLECTION}

All women were recruited at the Department of Obstetrics and Gynaecology at the Jessop Wing Hospital for Women, Sheffield. Informed consent was obtained from all patients and approved by the Leeds Bradford Local Research Ethics Committee (Ref No. 12/YH/0229). Lower segment myometrial biopsies were taken from healthy women undergoing elective cesarean sections at term ( $n=45$, age $16-43$, gestation $37-40$ weeks) as described previously. Myometrial smooth muscle cell cultures were then subsequently generated as detailed in Phaneuf et al. (1993).

\section{IMMUNOHISTOCHEMICAL STAINING FOR THE MaXiK AND L-TYPE $\mathrm{Ca}^{2+}$ CHANNELS}

A single myometrial biopsy was used to generate each individual primary culture. Myometrial cells were cultured in a 6-well plate for 2-4 passages. Control cells were human oral fibroblasts (a kind gift from Dr. Vanessa Hearnden, Human Nutrition Unit, University of Sheffield). Upon reaching $80 \%$ confluence the cells were harvested from two matched cultures from the same biopsy.
One of these underwent immunohistochemistry and the second, RNA extraction. For immunohistochemistry, cells were fixed in $4 \%(\mathrm{w} / \mathrm{v})$ paraformaldehyde overnight at $4^{\circ} \mathrm{C}$. Prior to staining the cells were washed 3 times for $5 \mathrm{~min}$ in PBS and permeabilized with PBS containing $1 \%(\mathrm{w} / \mathrm{v})$ BSA endogenous peroxidase was quenched with $3 \%(\mathrm{v} / \mathrm{v})$ hydrogen peroxide for $10 \mathrm{~min}$. Staining was then carried out using the Vectastain ${ }^{\circledR}$ Elite ABC Kit according to the manufacturer's instructions. Cells were stored in PBS and images capture using a Nikon DS-Fil Microscope camer and Olympus CKX41 microscope.

\section{COLLAGEN GEL CONTRACTION ASSAY}

Human myometrial cells were included in collagen gels as detailed in Ngo et al. (2006) Briefly, confluent cells were harvested and resuspended in DMEM with D-valine. A type-I collagen solution $(3 \mathrm{mg} / \mathrm{ml}$ in $0.1 \%$ acetic acid) was adjusted to $\mathrm{pH} 7.2$ with $1 \mathrm{M} \mathrm{NaOH}$. The final concentration of collagen was $1.5 \mathrm{mg} / \mathrm{ml}$ to which myometrial cells were then added. The collagen gel:cell suspension was incubated in a 24 well plate for $20 \mathrm{~min}$ to allow gelling, then $0.5 \mathrm{ml} \mathrm{DMEM}$ with $\mathrm{D}$-valine medium was added over the collagen lattice.

Upon reaching $80 \%$ confluence the collagen gel was dissociated from the sides and bottom of the well. Then the plate was gently swirled to ensure the gel was free floating. Myometrial cells were then stimulated with $10 \mathrm{ng} / \mathrm{ml}$ TNF or $2 \mu \mathrm{g} / \mathrm{ml} \mathrm{TSA}$, or left un-stimulated. PHM1-31 cells were used as contraction-positive controls while HEK293 cells were used as contraction negative controls (Chevillard et al., 2007; Fitzgibbon et al., 2009). At predetermined time points the plate was removed from the incubator for image capture and acquisition (SynGene GBox Chemi-16 gel documentation system). Subsequent surface area quantification was undertaken using GeneTools Version 4 quantification software (SynGene, Cambridge UK). Briefly, this software was used to trace the outline of the gel at each time point and the mean pixel area calculated. The surface area at each time point is reported as a percentage of the initial gel surface area in that well, a decrease in surface area indicates contraction whilst an increase indicates relaxation.

\section{RNA EXTRACTION AND cDNA SYNTHESIS}

On reaching $90 \%$ confluence the cells were stimulated with $10 \mathrm{ng} / \mathrm{ml} \mathrm{TNF}$ for $1 \mathrm{~h}$ or $2 \mu \mathrm{g} / \mathrm{ml}$ TSA for $24 \mathrm{~h}$ with nonstimulated cells serving as a control. RNA was extracted from the cells using the EZRNA extraction kit (Geneflow Ltd. Staffordshire, UK) according to the manufacturer's instructions. The extracted RNA was quantified using the nanophotometer (Implen, Germany). cDNA synthesis was performed using iScript cDNA synthesis Kit (Bio-Rad; Hertfordshire UK) according to the manufacturer's guidelines. One microlitre of cDNA was used per PCR.

\section{POLYMERASE CHAIN REACTION}

PCR was carried out using PCR Master Mix (Promega; Southampton UK) according to the manufacturer's guidelines. For characterization of the primary cultures Actin $\alpha 2$ (NM_001141945.1) was chosen as an indicator of the presence of myocytes and Thy-1 cell surface antigen (NM_006288.3), as 
an indicator of the presence of fibroblasts. Primers were designed to amplify each of the RNAs (Table 1) PHM1 RNA was used as a positive control for actin $\alpha 2$ and a negative control for Thy-1, Fibroblast RNA was used as a positive control for Thy-1.

For the detection of channel splice variants within the primary cultures, primers were selected to cover an un-spliced region in each channel and also to cover a number of spliced regions in each channel. The individual PCR primer sequences are detailed in Table 1; individual splice variant-specific PCR conditions are listed in Table 2. PCRs were performed using a Sensoquest Labcycler (Geneflow Ltd., Staffordshire, UK). All channel RTPCR reactions were done simultaneously and with the GAPDH

\section{Table 1 | Sequences of primers employed in RT-PCR experiments in this study.}

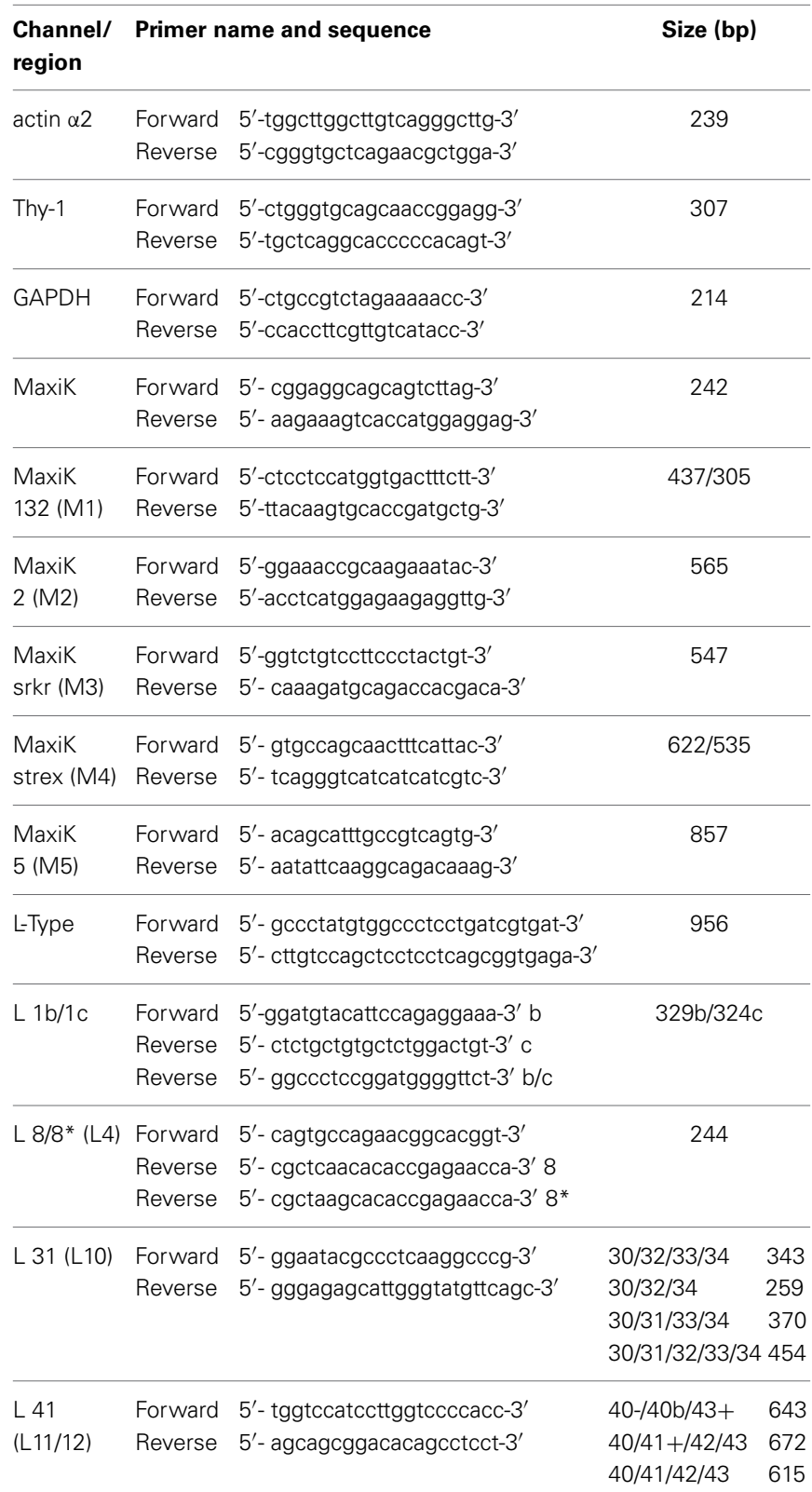

control serving experiments for both MaxiK and LTCC channels. Consequently Figure 3D is duplicated in Figure 4D purely for this reason. The PCR products were analyzed by agarose gel electrophoresis. The separated DNA fragments were visualized using UV light (wavelength $310 \mathrm{~nm}$ ). Images were captured using the SynGene GBox Chemi-16 gel documentation system. Subsequent band quantification and gel calibration with DNA ladders (LowRanger Ladder, 2000-100 bp Geneflow Ltd. Staffordshire, UK) was undertaken using GeneTools Version 4 quantification software (SynGene, Cambridge UK).

\section{ANALYSIS OF PCR PRODUCTS}

After manual band quantification of the splice variant PCR analysis was performed as follows: first each individual sample set and PCR reaction were analyzed separately. Within each sample set and PCR reaction the un-stimulated sample was quantified as $100 \%$ the stimulated samples for the matching PCR reactions were then quantified as a percentage of the un-stimulated reaction. The exception to this, however, was quantification of the LTCC exon- 8 variant after TSA treatment. Since unstimulated cultures and those exposed to TNF did not express exon- 8 in our experiments, we have needed to show the data as arbitrary units as we cannot give data as a percentage increase from a zero value. All experiments were repeated between three and eight times and results are expressed as the mean $\pm S D$. Each repeat represented one individual myometrial biopsy. All data analyses were conducted on GraphPad Prism Version 5.02 (GraphPad Software, San Diego, California) where a one way ANOVA for matched samples with Dunett's post-test was performed to compare the individual stimulations against the un-stimulated control; $\mathrm{p}<0.05$ was considered statistically significant.

\section{RESULTS}

\section{CHARACTERIZATION OF PRIMARY CULTURES}

Our aim was to determine if TNF and TSA exerted their effect on myometrial smooth muscle contractility via the regulation of the expression of either the MaxiK and LTCCs or their splice variants. As a first step we characterized the primary cells derived from the biopsies to verify their suitability as samples for this purpose.

\section{Table 2 | Reaction conditions employed for all channel RT-PCR} experiments in this study.

\begin{tabular}{lccc}
\hline $\begin{array}{l}\text { Channel/ } \\
\text { region }\end{array}$ & $\begin{array}{c}\text { Annealing } \\
\text { temp. }\left({ }^{\circ} \mathbf{C}\right)\end{array}$ & $\begin{array}{c}\text { Duration of denature/ } \\
\text { anneal/elongation } \\
\text { cycle (seconds) }\end{array}$ & $\begin{array}{c}\text { Number of } \\
\text { cycles }\end{array}$ \\
\hline MaxiK & 52 & $20 / 45 / 60$ & 35 \\
MaxiK 132 (M 1) & 54 & $25 / 30 / 45$ & 35 \\
MaxiK 2 (M 2) & 50 & $25 / 30 / 45$ & 35 \\
MaxiK srkr (M 3) & 54 & $25 / 30 / 45$ & 35 \\
MaxiK strex (M 4) & 52 & $25 / 30 / 45$ & 35 \\
MaxiK 5 (M 5) & 53 & $25 / 30 / 45$ & 35 \\
L-Type & 63 & $30 / 60 / 30$ & 40 \\
L 8/8* (L4) & 59 & $25 / 30 / 45$ & 40 \\
L 31 (L10) & 59 & $25 / 30 / 45$ & 40 \\
L 41 (L11/12) & 59 & $25 / 30 / 45$ & 40
\end{tabular}


To this end we first established that our primary cells cultures consisted primarily of myocyte cells rather than fibroblasts. This was done by measuring the expression of Actin $\alpha 2$, a smooth muscle actin and Thy-1 a cell surface antigen expressed specifically from fibroblasts. An immortalized human myometrial cell line PHM1-31 was used as a smooth muscle positive control and oral fibroblasts as a fibroblast cell positive control. PCR using Thy-1, showed only faint bands (Figure 1A) in the PHM131 cell line and the primary cultures indicting there is only a low level of fibroblast or myofibroblast contamination. In contrast to this and as expected, the fibroblast culture showed a much more intense Thy-1 band (Figure 1A). All cultures gave an intense band when Actin $\alpha 2$ primers were employed in the PCR indicating the presence of smooth muscle cells (Figure 1B). Importantly, within the primary myocyte cultures the level of fibroblast contamination was found to vary between 1.2 and $11 \%$ which is comparable with the immortalized PHM1-31 cell line (Figure 1C).

\section{CHANNEL IMMUNOCYTOCHEMISTRY}

Culturing of primary cells has been shown to be able to affect certain functional properties of the cells such as the loss of steroid receptors (Berns et al., 1985; Tyagi et al., 2006). The effect of culturing on ion channel expression is unclear. Consequently it was important to determine if the primary myocyte cultures expressed the MaxiK and LTCCs. Immunocytochemistry demonstrated that both channel types remain present in primary myometrial cell cultures (MaxiK-Figure 1D, Panel I; LTCC - Dig. 1D, Panel II. Antiserum controls Figure 1D, Panels III-IV). Interestingly, intense nuclear staining was observed for both channels although the significance of this remains unclear at present.

\section{PRIMARY MYOCYTE CONTRACTILITY}

The final step in characterizing the primary cultures was determining if they retained the ability to contract. A collagen contraction assay was used for this purpose. Primary cells were embedded within collagen gels and either left un-stimulated or stimulated with TNF or TSA. PHMI-31 cells and HEK293 cells left un-stimulated or stimulated with TNF or TSA were used as positive and negative controls respectively. The area of the gels was recorded to determine what effects the stimulants had on the contractility of the cells. A reduction in the surface area of the gel after the gel was released was an indication that the cells retained smooth muscle tone, i.e., the resistance to passive stretch during resting state. Upon the release of the gels from the sides of the well there was a visible reduction in the size of the gels containing both primary cells and PHM1-31 cells, there was no reduction in gel size seen for the HEK293 cells. This demonstrates that the primary cells still retain contractile tone. The amount of contraction was calculated as the inverse of the gel size after release expressed as a percentage of the original gel size (Figure 2A).

In the collagen gels containing HEK293 cells, no contraction was observed upon treatment with TNF (Figure 2B). In contrast to this, however, when collagen embedded PHM1-31 or primary cells, where stimulated with TNF, there was a significant reduction in gel surface area afterwards suggesting that TNF was inducing cell contraction (Figures 2B,C). Significantly, an increase in gel

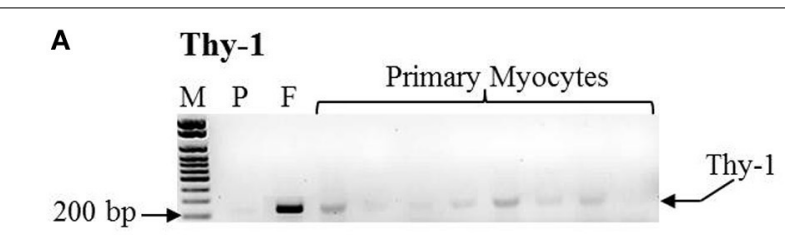

B Actin- $\alpha 2$

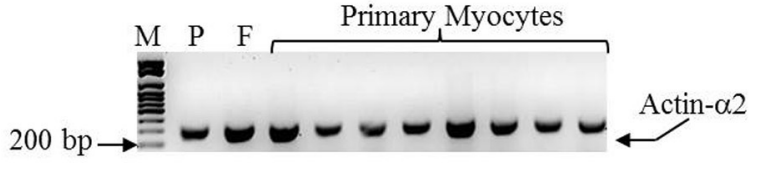

C Composition of Primary Cell Cultures

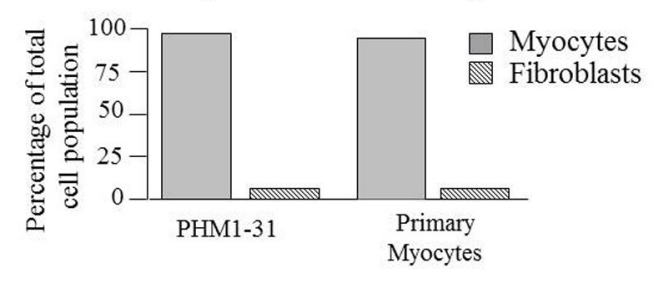

D Channel Immunocytochemistry

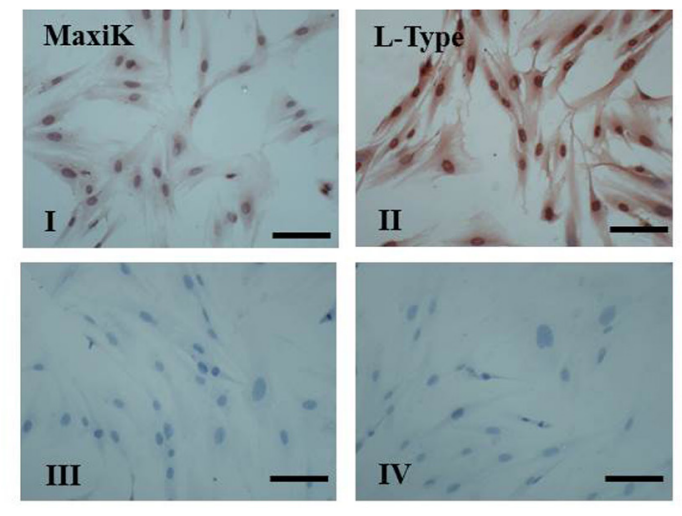

FIGURE 1 | Primary myometrial cell cultures are composed primarily of myocytes and express both the MaxiK and L-type $\mathrm{Ca}^{2+}$ channels. RNA from primary myometrial cell cultures was extracted and amplified by PCR. (A) Primary myometrial cell cultures express a low level of Thy-1 mRNA indicating a low level of Fibroblasts in the culture. (B) Primary myometrial cell cultures express a high level of Actin- $\alpha 2$ mRNA indicating a high level of myocytes in the culture. Manual quantification of the relative band intensities was used to confirm the presence of myocytes and to estimate the level of fibroblast contamination. (C) Primary myometrial cell cultures are comprised of 88-98\% myocytes (gray bar) and between 1.2 and $11 \%$ fibroblasts (striped bar) which is comparable to the PHM1 cultures. (D) Myometrial cells were staining using antibodies specific to MaxiK (I) and L-Type (II) channels. Controls excluded primary (III) and secondary antibody (IV). Dark red/brown staining denotes specific staining of the protein of interest (scale bar is $100 \mu \mathrm{m}$ ).

surface area was observed when cultures were treated with TSA indicating that it induced a loss of basal tone in the collagenembedded myocyte cultures although this did not reach statistical significance (Figures 2B,C). 
A

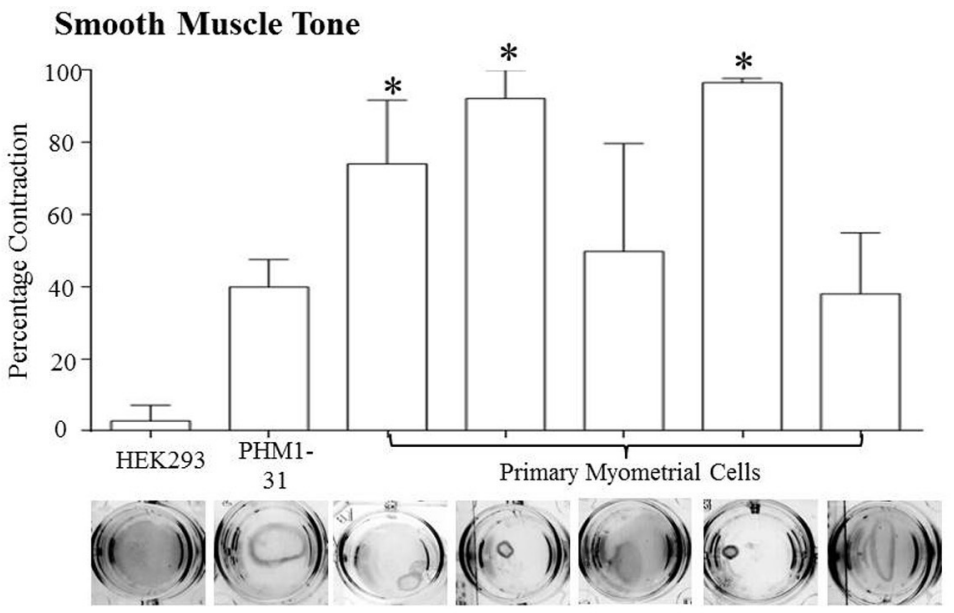

B Collagen Gel Contraction
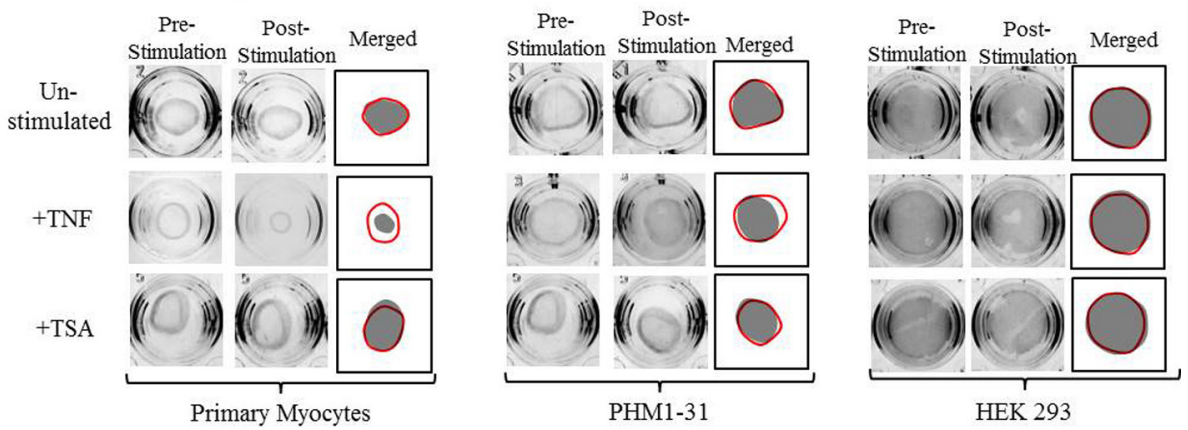

C

Percentage Contraction

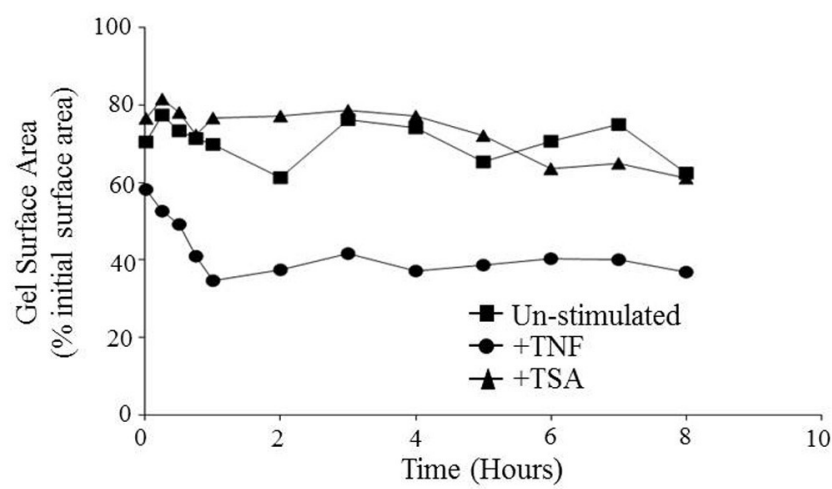

FIGURE 2 | Primary myometrial cell cultures retain smooth muscle tone and the ability to contract. (A) The reduction of the surface area of the collagen after release demonstrates the retention of smooth muscle tone in the primary myometrial cell cultures. In the more confluent cultures this reduction in gel size becomes significant $\left({ }^{*} p<0.05\right)$ There is no reduction in gel size in the HEK293 cultures. (B) Depiction of the maximum gel contraction for each culture after stimulation. (C) Depiction of the change in collagen gel surface area of the primary cells over the course of the experiment.

\section{MaxiK SPLICE VARIANTS ARE EXPRESSED IN NON-LABORING TERM PREGNANT MYOMETRIUM AND ARE DOWN-REGULATED BY TRICHOSTATIN-A}

In our previous studies, we have reported differential effects of both TNF and TSA on signaling pathways in primary myometrial myocytes (Chapman et al., 2005; Webster et al., 2013). In the present study we sought to determine if such compounds could also influence expression of the MaxiK mRNA. Total cellular RNA was amplified using primers specific for a region within MaxiK that is conserved in all splice variants. As such, this would give an indication of overall expression of the mRNA for this channel and how TNF and TSA affected this. Figure 3A demonstrates 


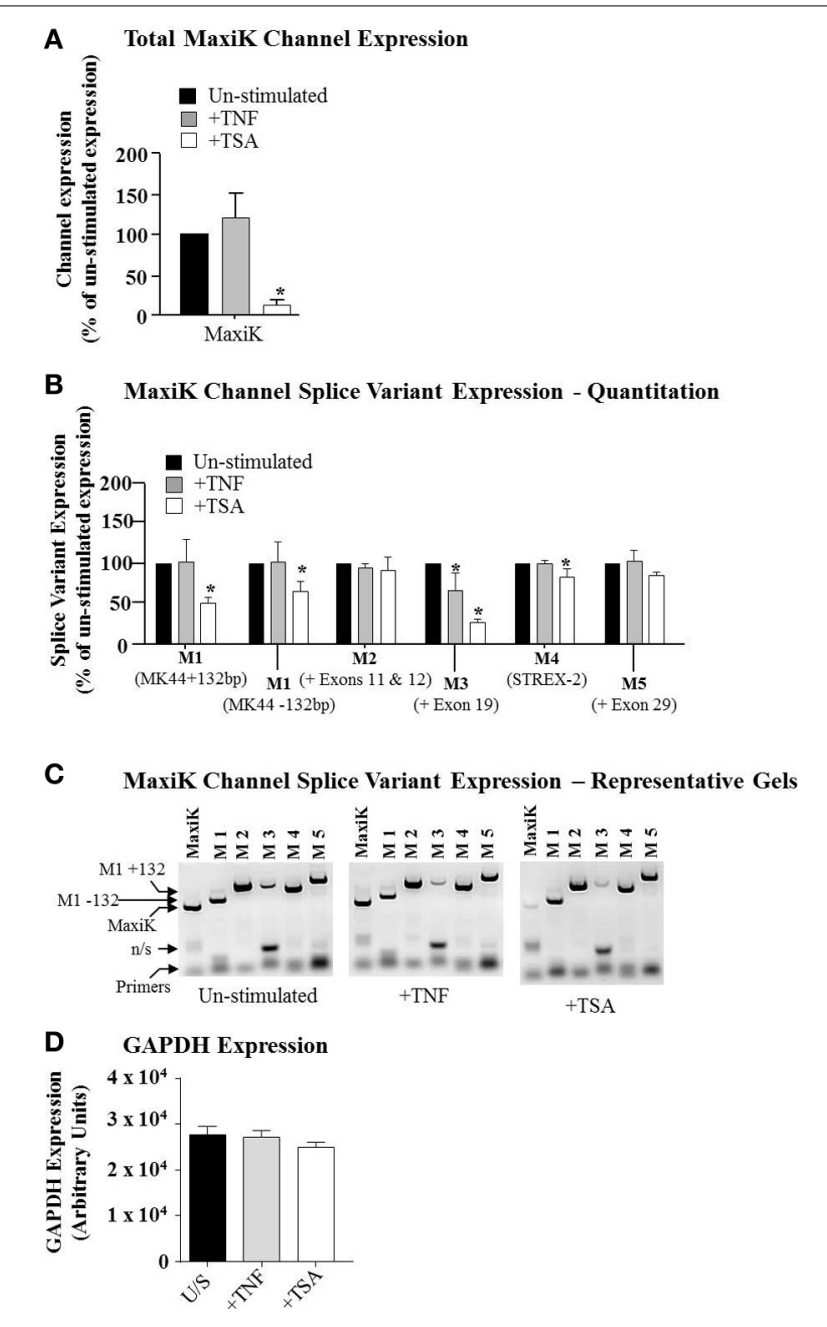

FIGURE 3 | The expression of the MaxiK channel and splice variants in primary myometrial cell cultures. Primary myometrial cell cultures were stimulated with $10 \mathrm{ng} / \mathrm{ml}$ TNF for $1 \mathrm{~h}, 100 \mathrm{ng} / \mathrm{ml}$ TSA for $24 \mathrm{~h}$ or left un-stimulated. RNA was extracted, reverse transcribed and amplified using channel and splice variant specific primers. Manual quantification of the relative band intensities were used to quantify the level of expression and this was expressed as a percentage of the un-stimulated expression. (A) TNF had no significant effect whilst TSA significantly reduced the expression of the MaxiK channel (* $p<0.05)$. (B) Primary Myometrial cell cultures express a range of MaxiK splice variants, TNF had no effect on splice variant expression and TSA significantly reduced the expression of the MK44 splice variant $\left({ }^{*} p<0.05\right)$. (C) Representative gel of the RT-PCR products. (D) Neither TNF nor TSA influenced the expression of GAPDH.

that TSA was seen to induce a significant reduction in expression of MaxiK mRNA (Figure 3A, white bar). In contrast to this, TNF was seen to induce an increase in total MaxiK mRNA expression but this did not reach significance (Figure 4A, gray bar). Neither treatment influenced the expression of GAPDH (Figure 3D).

The MaxiK channel is documented to have a number of splice variants (Curley et al., 2004). Consequently, it was important to determine if the expression of such variants also occurred in primary human myometrial myocytes. Moreover, it was also salient to determine which of these variants were sensitive to
TSA treatment. Total cellular RNA was amplified using primers specific for the different splice sites published for the MaxiK open reading frame. Primary myometrial myocytes were seen to express a number of different splice variants M1 (including both the MK44 variants which either express a $132 \mathrm{bp}$ insert between exons 1 and 2 or have this insert omitted); M2 (+exon 11 and 12); (M3 (+ exon 19); M4 (STREX-2); and M5 (+exon 29)

\section{(Figures 3B,C).}

When cultures were subsequently exposed to either TNF or TSA, TSA significantly decreased the expression of the MK44 splice variant in comparison to un-stimulated cultures and the ratio between the MK44 variant containing the $132 \mathrm{bp}$ insert and the variant lacking this insert was also significantly reduced with TSA stimulation. TNF had no effect on either the expression of the MK44 variant or on the ratio between the insert and insertless forms of the variant (Figure 3B). TNF did, however, induce repression of the M3 (+ exon 19) variant. We also observed a similar TSA-induced repression of both the M3 (+ exon 19) variant and the M4 (STREX-2) variant (Figure 3B). The observed reduction in STREX-2 levels, while statistically significant, was, however, small in magnitude and the relevance of this remains unclear at present. Representative gels of each treatment are illustrated in Figure 3C. No change in GAPDH was observed with either treatment (Figure 3D).

\section{L-TYPE $\mathrm{Ca}^{2+}$ CHANNEL SPLICE VARIANTS ARE EXPRESSED IN NON-LABORING TERM PREGNANT MYOMETRIUM AND ARE DOWN-REGULATED BY TRICHOSTATIN-A}

Next, we determined if these compounds also influence expression of the LTCC mRNA. Total cellular RNA was amplified using primers specific for a region within LTCC that is conserved in all splice variants. As such, this would give an indication of overall expression of the mRNA for this channel and how TNF and TSA affected this. Figure 4A demonstrates that TSA was seen to induce a reduction in expression of LTCC mRNA but this did not reach significance (Figure 4A, white bar). In contrast to this, TNF was seen to induce a significant increase in total LTCC mRNA expression (Figure 4A, gray bar). Neither treatment influenced the expression of GAPDH (Figure 4D).

The LTCC is documented to have a number of splice variants (Tang et al., 2004). Consequently, it was important to determine if the expression of such variants also occurred in primary human myometrial myocytes. Moreover, it was also salient to determine which of these variants were sensitive to TNF treatment. Total cellular RNA was amplified using primers specific for the different splice sites published for the LTCC open reading frame. Primary myometrial myocytes were seen to express a number of different splice variants (Figures 4B,C). Briefly, the myocytes express the splice site 4 variant containing exon $8^{*}$ this variant has lower DHP sensitivity, more rapid activation and slower deactivation kinetics than the alternative exon 8-containing variant (Soldatov, 1992). Two splice site 10 variants were observed one containing exons 30 , $31,32,33$ and 34 and a second containing exons 30, 31, 33 and 34 these two variants affect the size and rigidity of the S3 to S4 linker segments. It has been suggested that shorter linker segments result in channels with slower gating kinetics while longer linking segments result in channels with faster gating kinetics (Perez-Reyes 


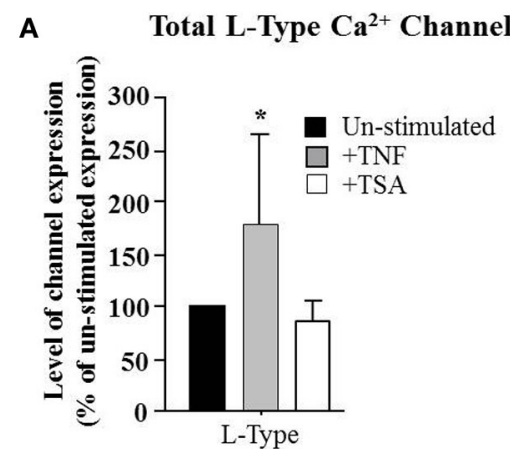

B L-Type $\mathrm{Ca}^{2+}$ Channel Splice Variant Expression - Quantitation

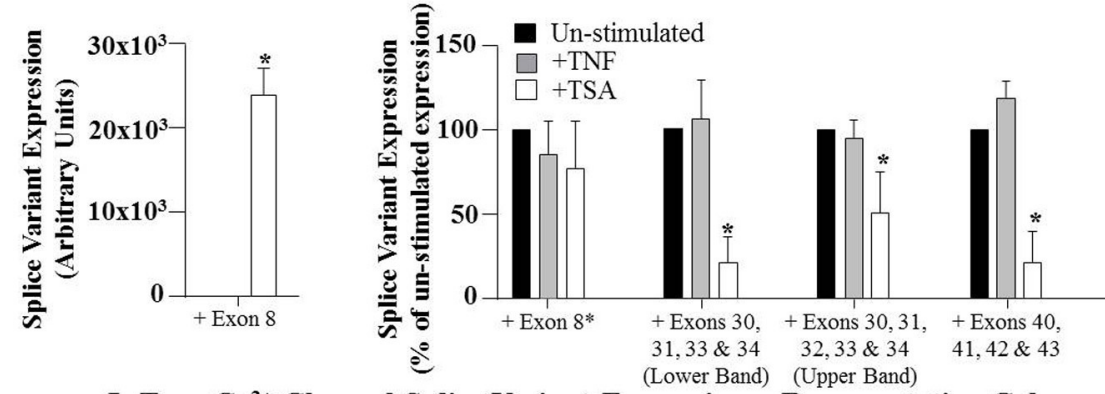

C L-Type $\mathrm{Ca}^{2+}$ Channel Splice Variant Expression - Representative Gels

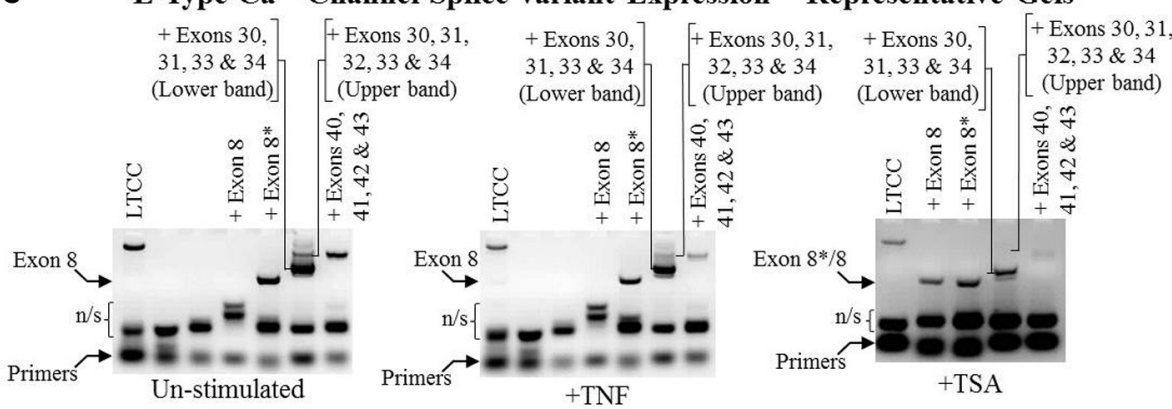

D

GAPDH Expression

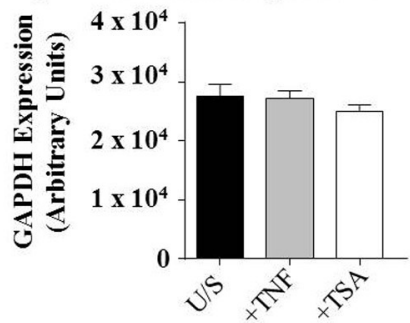

FIGURE 4 | The expression of the L-Type $\mathrm{Ca}^{2+}$ channel and splice variants in primary myometrial cell cultures. Primary myometrial cell cultures were stimulated with $10 \mathrm{ng} / \mathrm{ml}$ TNF, $100 \mathrm{ng} / \mathrm{ml}$ TSA or left unstimulated. RNA was extracted, reverse transcribed and amplified using channel and splice variant specific primers. Manual quantification of the relative band intensities were used to quantify the level of expression and this was expressed as a percentage of the un-stimulated expression. (A) TNF significantly increased the expression of the L-Type $\mathrm{Ca}^{2+}$ channel whilst TSA had no significant effect $(* p<0.05)$. (B) Primary myometrial cell cultures express a range of L-Type $\mathrm{Ca}^{2+}$ channel splice variants. TSA significantly induced expression of the exon-8 variant (Left Panel; ${ }^{*} p<0.05$ ) whilst also reducing expression of a number of other splice variants (Right Panel; $* p<0.05$ ). TNF had no effect on splice variant expression. (C) Representative gel of the RT-PCR products. (D) Neither TNF nor TSA influenced the expression of GAPDH. et al., 1990; Yang et al., 2000). Of the two variants found in the primary cells the one containing 30,31, 33 and 34 is the shorter variant and was found in approximately $80 \%$ of the RNA. Finally the splice site 11 variant containing exons 40, 41, 42 and 43 was also observed.
When cultures were subsequently exposed to either TNF or TSA, TSA resulted in the novel expression of LTCC splice variants utilizing exon 8 (Figure 4B; Left Panel). Un-stimulated cells and those treated with TNF exclusively utilize exon $8^{*}$; after treatment with TSA, however, approximately $45 \%$ of the RNA 
expressed contained the exon 8 variant. The utilization of exon 8 leads to the expression of channels with higher DHP sensitivity and slower activation and more rapid deactivation curves (Figures 4B,C).

TSA also resulted in the down regulation of both forms of the splice site 10 variant while TNF had little effect (Figures 4B,C). The effect of TSA is what would be expected as the faster gating kinetics of the longer form would mean that it would take less time for the channels to fully open and hence would increase the influx of calcium and would promote contraction. The effect of a decreased expression of this variant would therefore lead to relaxation (Figures $4 \mathrm{~B}, \mathrm{C}$ ). Neither treatment influenced the expression of GAPDH (Figure 4D).

TSA also resulted in a down regulation of the splice site 11 variant. All the treatment groups expressed the same variant at splice site 11, this variant contained exons 40, 41, 42 and 43 with no additions or deletions. After TSA treatment the expression of this variant was significantly reduced, there was, however, no alternative variant expressed (Figures 4B,C).

\section{DISCUSSION}

At the end of pregnancy the myometrium switches from a state of electrical quiescence seen throughout gestation to the contractile state characteristic of parturition which then culminates in the birth of the baby. Although the molecular basis of contraction is understood, the triggers which move the myometrium from the quiescence to the contractile state seen at parturition remain to be defined.

Myometrial electrical quiescence state is characterized by slow wave potentials where the membrane potential cycles between depolarizations and repolarizations without reaching the threshold level for action potential generation (Parkington et al., 1999). However, in myometrium obtained at term gestation, slow wave potentials become frequent and synchronized action potentials during which the membrane potential rapidly rises and falls, causing the muscle to contract (Wilde and Marshall, 1988; Khan et al., 1993b).

Both the quiescent state and the coordination of contractions are thought to be mediated through mechanisms that involve ion channels. Therefore we postulate that the balance between the MaxiK channel and the LTCC is of key significance in the switch from electrical quiescence to activation and synchrony of contractions.

\section{THE MaxiK CHANNEL}

This research has demonstrated that term myometrium contains a number of different MaxiK channel splice variants including MK44 and STREX-2. The MK44 variant comprises a $132 \mathrm{bp}$ insertion, which has previously been described by Korovkina et al. (2001). Variants containing the insert have a diminished calcium and voltage sensitivity; the inserted sequence itself has been shown to contain both a phosphorylation and a myristylation site. This variant can also undergo proteolytic cleavage after which the $\mathrm{N}$ terminal is located on the membrane while the $\mathrm{C}$-terminal is held with the endoplasmic reticulum. The $\mathrm{C}$ terminal is released after release of calcium from sarcoplasmic reticulum based stores and re-constitutes with the N-terminus on the membrane. The presence of this variant shows that there are MaxiK channels with diminished calcium and voltage sensitivity within the myometrium and is in agreement with electrophysiological results from our studies that demonstrate altered calcium and voltage-sensitivity of the MaxiK channel in laboring human myometrium (Khan et al., 1993b, 1997). Curley et al. (2004) demonstrated that the variant containing the $132 \mathrm{bp}$ insert increased in laboring myometrium; this, however, was not evident in our study.

The MaxiK Splice site 4 consists of the differential utilization of exons 22 and 23. The variant present in the myocytes contained both exons 22 and 23 and so is the STREX-2 variant (Xie and McCobb, 1998). The STREX-2 variant has increased mechanosensitivity and inhibition by hypoxia. Interestingly the STREX insert also contains a cAMP dependant Protein Kinase A (PKA) consensus motif which when phosphorylated facilitates membrane depolarization hence promoting the contractile phenotype of the cells.

\section{THE L-TYPE $\mathrm{Ca}^{2+}$ CHANNEL}

We have demonstrated that term myometrium expresses procontractile splice variants of the LTCC, including Splice site 4 containing exon $8^{*}$, Splice site 10 containing exons 30,31,32 and 34 and splice site 11 containing exons 40, 41, 42 and 43. LTCC Splice site 4 is comprised of the mutually exclusive use of exon 8 or $8^{*}$. The PCR results show that unstimulated term myocytes express exon $8^{*}$. The expression of $8^{*}$ results in a channel with decreased DHP sensitivity, rapid activation and slow deactivation kinetics (Goodwin et al., 1999). The kinetics of this channel variant would promote contraction.

The LTCC Splice site 10 is a complex site which involves the differential usage of exons $30,31,32,33$, and 34 which result in different length of linking loop between IV S2 and S4. The uterine myocytes express two forms of this variant- a longer one containing exons $30,31,32,33$, and 34 and a shorter one containing exons 30, 31, 33, and 34. The longer form containing exon 32 correlates well with SVI3B, which has been shown to have increased excitation-contraction coupling (Huang et al., 2006) and so the less abundant expression of this form would decrease the excitability of the cell and promote relaxation.

The LTCC Splice site 11 encompasses exons 40, 41, 42, and 43 , variants in this region include use of exon 40A (exon40 $19 \mathrm{bp}$ ), $+125 \mathrm{bp}$ (exon 40B), exon $41 \mathrm{~A}$ and exon $43+132 \mathrm{bp}$ or the use of exons 40,41, 42, and 43 (Soldatov, 1994). The additional $19 \mathrm{bp}$ found in exon 40 in comparison to exon $40 \mathrm{~A}$ are thought to modulate the tethering of calmodulin to the C-terminal and impact on the calcium dependant inactivation of the channel (Gerhardstein et al., 2000). The splice site 11 variant found to be expressed in the myocytes contained exons 40, 41, 42 , and 43 . The variant found at splice site 11 is the same variant Kepplinger et al. reported and named $\alpha 1 \mathrm{C}_{77}$ (Kepplinger et al., 2000 ). Calcium-dependant inactivation was found to be the highest in the $\alpha 1 C_{77}$ variant (Soldatov et al., 1997). Also, this variant was found to be more efficiently targeted to the cell membrane and had higher conductance and open probability than other variants at this site (Kepplinger et al., 2000). The expression of this variant would push the cell toward a more contractile state. 
Following TSA stimulation there was an overall reduction in the expression of variants at splice sites L10 and L11/12, there are two explanations for this, firstly the reduction in total LTCC RNA as a result of TSA treatment may result in an apparent reduction in this particular splice variant. We have compensated for this possibility by expressing the level of splice variant expression as a percentage of the total channel expression within the same treatment group. The second possibility is that the RNA is truncated (Snutch et al., 1991). There are two splice variants which have not been looked at in this study, of specific interest here is the possibility of a 4 nt insertion into the $5^{\prime}$ end of exon 3 . This insertion shifts the reading frame giving rise to a premature stop codon and resulting in the production of only the $\mathrm{N}$ terminus of the LTCC (Snutch et al., 1991; Soldatov, 1992). If this is the reason for the reduction in the splice site L10 and L11/12 variants then it would appear that TSA causes an increase in truncated protein.

The possible presence of a truncated LTCC comprising only the $\mathrm{N}$-terminal domain is interesting as the $\mathrm{C}$-terminus has been shown to exert an inhibitory role over the channel (Hell et al., 1996) and so a functional channel lacking the C-terminus will open more readily and support contraction. It is also possible that the putative truncated channel is silent. Either way this is certainly worthy of further investigation in understanding the control of myometrial contractility. These findings provide experimental evidence that toward the end of gestation, myometrium is primed ready to contract based on the channel variants present. Further work, however, directly comparing pregnant, non-laboring human myometrium to actively laboring tissue would be required to confirm this thesis.

\section{THE ACTION OF TNF}

The initiation of the contractile state seen at parturition is characterized by an influx of pro-inflammatory cytokines such as IL-1 $\beta$, IL-6, and TNF (Romero et al., 1992; Opsjln et al., 1993; Keelan et al., 1999; Osman et al., 2003). This influx leads to the activation of $\mathrm{NF} \kappa \mathrm{B}$ which in turn promotes the expression of procontractile genes (Mendelson, 2009) as well as repressing those genes promoting myometrial quiescence such as Gas (Chapman et al., 2005; Webster et al., 2013). As such, our observations from the mono-layer contraction studies demonstrating the contractile action of TNF and the pro-relaxant function of TSA are in broad agreement with the published data on the effects of TNF and TSA on contractility of isolated smooth muscle strips (Sadowsky et al., 2006; Fitzgibbon et al., 2009; Europe-Finner et al., 2013; Webster et al., 2013).

Whilst studying the function of the individual TNF receptors, TNFR1 and TNFR2, was outside the scope of this study, it is important to consider which receptor the TNF signal utilizes. For example, potassium channels can directly interact with TNFR1, and in airway smooth muscle it was shown that a change in contractile response induced by TNF was via TNFR1 signaling (Amrani et al., 2000; Tliba et al., 2004; Bronstein-Sitton, 2006; Wang et al., 2011). This study within the myometrium suggests that TNF signaling is through TNFR1 (Leroy et al., 2007). Finally, a study in cardiac myocytes demonstrates complex cross talk between TNFR1 and TNFR2 to control calcium transients (Defer et al., 2007).
TNF is a potent activator of NFאB (Cookson and Chapman, 2010); interestingly, the promoter of the MaxiK channel $\alpha$ subunit contains NFKB motifs which are enriched with the RelAcontaining NFKB dimers after $1 \mathrm{~h}$ exposure to TNF (Cookson, 2010). Together, these data suggest that the myometrium may respond to such pro-inflammatory agents and permit transient changes in the pattern of channel expression associated with cellular exposure to cytokines (Dhulipala and Kotlikoff, 1999; Lu et al., 1999).

In terms of calcium flux, using hippocampal neurons, TNF stimulation has been shown to increase calcium current density by around $20 \%$ and this increase can be attributed to an increase in LTCC current (Furukawa and Mattson, 1998). Furukawa et al. went on to show that this increase was dependant on NFKB activation as inhibition of $\mathrm{NF} \kappa \mathrm{B}$ resulted in repression of these calcium current increases.

Calcium sensitization has also been suggested as playing a role in the switch from quiescence to contractility. Calcium sensitization is the phenomenon by which a given concentration of intracellular calcium results in a larger than expected force of contraction (Ratz et al., 2005; Arthur et al., 2007; Wray, 2007). TNF has been shown to enhance $\mathrm{Ca}^{2+}$ responsiveness tenfold in airway smooth muscle. It has been shown that TNF may cause this increased $\mathrm{Ca}^{2+}$ sensitivity through inducing increased $\mathrm{IP}_{3}$ turnover and hence increased release of $\mathrm{Ca}^{2+}$ from intracellular stores.

In this study TNF was shown to significantly increase the transcription of the MaxiK channel. This would initially seem at odds with TNF promoting contraction, however, an up-regulation of transcription does not always correspond to an up-regulation of protein levels. Kim et al. demonstrated this lag between MaxiK RNA and protein up-regulation in chick cochlear development and determined that it was due to delays in protein synthesis and trafficking/scaffolding of the channel subunits (Kim et al., 2010). A similar delay may be occurring in the myometrium.

The expression of the MaxiK STREX-2 variant was unchanged with TNF $\alpha$ stimulation, however, Gas is known to decrease during labor (Europe-Finner et al., 1994) and as a result of this the level of PKA would decrease. This decrease in PKA would result in decreased phosphorylation of the MaxiK channel, but any phosphorylation in the presence of STREX-2 would facilitate membrane depolarization and support contraction (Tian et al., 2004). This support of contraction would decrease as the levels of PKA decrease. Hypothetically this could lead to support for contraction while the PKA levels are high at the start of parturition, then a gradual switch to support quiescence as the PKA levels drop leading to the termination of parturition.

TNF also resulted in a significant up regulation of the LTCCs, while the overall splice variant profile was unchanged.

\section{THE ACTION OF TSA}

Trichostatin A is a potent histone deacetylase inhibitor (HDACi) which results in an increase in histone acetylation and can alter gene expression by preventing DNA transcription factors from accessing the DNA. It can also induce global protein acetylation (Spange et al., 2009). Studies have shown that TSA also has a potent inhibitory effect of myometrial contractility strips 
(Lu et al., 1999; Moynihan et al., 2008; Europe-Finner et al., 2013; Webster et al., 2013), although the relaxant effect induced by TSA could be overcome by pro-inflammatory agents such as TNF (Webster et al., 2013). While TSA is a functional HDACi it can, of course, modulate non-histone proteins acetylation too and this has been reviewed in the myometrium and broader research fields (Spange et al., 2009; Europe-Finner et al., 2013). Indeed, the non-nuclear lysine deacetylase (KDAC) KDAC8 modified Hsp20 acetylation profile in such a manner that myometrial contractility was subsequently inhibited (Karolczak-Bayatti et al., 2011). Consequently, we speculate that in myometrial cells, such putative global acetylation, coupled with chromatin-based effects, could influence the balance of channel splice variant formation.

The transition of the myometrium from the quiescent state to the contractile state seen at parturition has been shown to be facilitated by the down regulation of the hCG/LH receptors. Moynihan et al. (2008) demonstrated that TSA promoted the transcriptional activation of the hCG/LH receptor gene and hence the maintenance of quiescence (Phillips et al., 2005; Moynihan et al., 2008). It has also been shown that the maintenance of myometrial quiescence is facilitated by the expression of Gas. Acetylation of the Gas promoter by Cyclic-AMP Response Element Binding Protein (CREB) Binding Protein (CBP) is necessary for its expression within the myometrium and therefore TSA may also act by preventing the deacetylation of the Gas promoter and hence promote the expression of $\mathrm{G} \alpha$ s and the maintenance of myometrial quiescence (Webster et al., 2013).

This research suggests a further possible mechanism for the pro-quiescent action of TSA via its effect on the expression of both the MaxiK and LTCCs. TSA resulted in the significant down regulation of both channels. After TSA stimulation the LTCC expressed variants containing exon 8 and those containing $8^{*}$, the presence of variants containing exon 8 would result in a less contractile phenotype. This reduction in MaxiK and LTCC transcription would result in cells much less responsive to calcium and so they would be much less contractile.

\section{CONCLUSION}

This research suggests that increasing TNF levels at parturition promote the increased calcium sensitivity of the myometrium. We suggest this occurs through expression of MaxiK channel variants which have both decreased calcium and voltage sensitivity, in conjunction with LTCCs displaying rapid activation, slow deactivation kinetics and increased excitation-contraction coupling within the myocytes (Soldatov et al., 1997; Goodwin et al., 1999; Korovkina et al., 2001; Tian et al., 2004; Huang et al., 2006).

The combined effect of TNF on IP3 turnover and subsequent release of $\mathrm{Ca}^{2+}$ from intracellular stores, the increase of transcription of the LTCCs and the expression of MaxiK channel variants with decreased calcium and voltage sensitivity may serve to tip the fine balance of the channels allowing action potentials to be generated and propagated across the tissue resulting in contraction.

TSA was seen to promote a relaxatory effect on the cells, this is appears to be through the significant reduction in transcription of both channels resulting in cells that are less excitatory. TSA has been suggested as a potential tocolytic due to its relaxatory effect on the myometrium. More work, however, is required to determine the combined effect of TSA and TNF on myometrial excitability and how these functions involve protein acetylation, within chromatin and globally within the cyctosol, before such HDAC and KDAC inhibitors could be used as successful clinical interventions for pre-term labor.

\section{FUNDING}

This work was funded by The Jessop Wing Small Grants Scheme (Grant Nos. OGN/13/01 to Sarah L. Waite and OGN/11/05 and OGN/12/02 to Neil R. Chapman).

\section{ACKNOWLEDGMENTS}

We would like to thank Prof. Nick Europe-Finner for his critical reviews of the manuscript prior to submission. We are grateful to the patients of the Jessop Wing, Sheffield Teaching Hospitals NHS Foundation Trust for participating in this study and to the clinical staff for obtaining samples.

\section{REFERENCES}

Aguilar, H. N., and Mitchell, B. F. (2010). Physiological pathways and molecular mechanisms regulating uterine contractility. Hum. Reprod. Update 16, 725-744. doi: 10.1093/humupd/dmq016

Amrani, Y., Chen, H., and Panettieri, R. A. (2000). Activation of tumor necrosis factor receptor 1 in airway smooth muscle: a potential pathway that modulates bronchial hyper-responsiveness in asthma? Respir. Res. 1, 49-53. doi: $10.1186 / \mathrm{rr} 12$

Anwer, K., Oberti, C., Perez, G. J., Perez-Reyes, N., McDougall, J. K., Monga, M., et al. (1993). Calcium-activated K+ channels as modulators of human myometrial contractile activity. Am. J. Physiol. 265, C976-C985.

Arthur, P., Taggart, M. J., and Mitchell, B. F. (2007). Oxytocin and parturition: a role for increased myometrial calcium and calcium sensitization? Front. Biosci. 12, 619-633. doi: 10.2741/2087

Bannister, J. P., Thomas-Gatewood, C. M., Neeb, Z. P., Adebiyi, A., Cheng, X., and Jaggar, J. H. (2011). Ca(V)1.2 channel N-terminal splice variants modulate functional surface expression in resistance size artery smooth muscle cells. J. Biol. Chem. 286, 15058-15066. doi: 10.1074/jbc.M110.182816

Berns, E. M., Brinkmann, A. O., Rommerts, F. F., Mulder, E., and van der Molen, H. J. (1985). Changes of oestrogen receptor levels in Leydig cells from mice and rats during culture. J. Steroid Biochem. 22, 293-298. doi: 10.1016/00224731(85)90428-5

Blencowe, H., Cousens, S., Oestergaard, M. Z., Chou, D., Moller, A. B., Narwal, R., et al. (2012). National, regional, and worldwide estimates of preterm birth rates in the year. (2010). with time trends since. (1990). for selected countries: a systematic analysis and implications. Lancet $379,2162-2172$. doi: 10.1016/S0140-6736(12)60820-4

Brainard, A. M., Miller, A. J., Martens, J. R., and England, S. K. (2005). Maxi-K channels localize to caveolae in human myometrium: a role for an actinchannel-caveolin complex in the regulation of myometrial smooth muscle K+ current. Am. J. Physiol. Cell Physiol. 289, C49-C57. doi: 10.1152/ajpcell.00399.2004

Bronstein-Sitton, N. (2006). K+ Channels and Cancer: Surprising New Discoveries, Pathways. Jerusalem: Alomone Labs.

Buxton, I. L., Singer, C. A., and Tichenor, J. N. (2010). Expression of stretchactivated two-pore potassium channels in human myometrium in pregnancy and labor. PLoS ONE 5:e12372. doi: 10.1371/journal.pone.0012372

Carney, S. A., Tahara, H., Swartz, C. D., Risinger, J. I., He, H., Moore, A. B., et al. (2002). Immortalization of human uterine leiomyoma and myometrial cell lines after induction of telomerase activity: molecular and phenotypic characteristics. Lab. Invest. 82, 719-728. doi: 10.1097/01.LAB.0000017499.51216.3E

Chang, H. H., Larson, J., Blencowe, H., Spong, C. Y., Howson, C. P., CairnsSmith, S., et al. (2013). Preventing preterm births: analysis of trends and potential reductions with interventions in 39 countries with very high human development index. Lancet 381, 223-234. doi: 10.1016/S0140-6736(12) 61856-X 
Chanrachakul, B., Broughton Pipkin, F., and Khan, R. N. (2004). Contribution of coupling between human myometrial beta2-adrenoreceptor and the $\mathrm{BK}(\mathrm{Ca})$ channel to uterine quiescence. Am. J. Physiol. Cell Physiol. 287, C1747-C1752. doi: 10.1152/ajpcell.00236.2004

Chapman, N. R., Smyrnias, I., Anumba, D. O., Europe-Finner, G. N., and Robson, S. C. (2005). Expression of the GTP-binding protein (Galphas) is repressed by the nuclear factor kappaB RelA subunit in human myometrium. Endocrinology 146, 4994-5002. doi: 10.1210/en.2005-0533

Chevillard, G., Derjuga, A., Devost, D., Zingg, H. H., and Blank, V. (2007). Identification of interleukin-lbeta regulated genes in uterine smooth muscle cells. Reproduction 134, 811-822. doi: 10.1530/REP-07-0289

Collins, P. L., Moore, J. J., Lundgren, D. W., Choobineh, E., Chang, S. M., and Chang, A. S. (2000). Gestational changes in uterine L-type calcium channel function and expression in guinea pig. Biol. Reprod. 63, 1262-1270. doi: 10.1095/biolreprod63.5.1262

Cookson, V. J., and Chapman, N. R. (2010). NF-kappaB function in the human myometrium during pregnancy and parturition. Histol. Histopathol. 25, 945-956. Available online at: http://www.hh.um.es/Abstracts/ Vol_25/25_7/25_7_945.htm

Cookson, V. J. K. W. (2010). Identifying Regulatory Gene Networks in the Myometrium Governed by NF-kappaB, Human Metabolism. Sheffield: University of Sheffield.

Costeloe, K. L., Hennessy, E. M., Haider, S., Stacey, F., Marlow, N., and Draper, E. S. (2012). Short term outcomes after extreme preterm birth in England: comparison of two birth cohorts in 1995 and 2006 (the EPICure studies). BMJ 345:e7976. doi: 10.1136/bmj.e7976

Curley, M., Morrison, J. J., and Smith, T. J. (2004). Analysis of Maxi-K alpha subunit splice variants in human myometrium. Reprod. Biol. Endocrinol. 2, 67. doi: 10.1186/1477-7827-2-67

Defer, N., Azroyan, A., Pecker, F., and Pavoine, C. (2007). TNFR1 and TNFR2 signaling interplay in cardiac myocytes. J. Biol. Chem. 282, 35564-35573. doi: 10.1074/jbc.M704003200

Dhulipala, P. D., and Kotlikoff, M. I. (1999). Cloning and characterization of the promoters of the maxiK channel alpha and beta subunits. Biochim. Biophys. Acta 1444, 254-262. doi: 10.1016/S0167-4781(98)00276-0

Diebold, R. J., Koch, W. J., Ellinor, P. T., Wang, J. J., Muthuchamy, M., Wieczorek, D. F., et al. (1992). Mutually exclusive exon splicing of the cardiac calcium channel alpha 1 subunit gene generates developmentally regulated isoforms in the rat heart. Proc. Natl. Acad. Sci. U.S.A. 89, 1497-1501. doi: 10.1073/pnas.89. 4.1497

Europe-Finner, G. N., Phaneuf, S., Tolkovsky, A. M., Watson, S. P., and Lopez Bernal, A. (1994). Down-regulation of $G$ alpha s in human myometrium in term and preterm labor: a mechanism for parturition. J. Clin. Endocrinol. Metab. 79, 1835-1839.

Europe-Finner, G. N., Taggart, M. J., and Karolczak-Bayatti, M. (2013). A role for cytoskeletal protein acetylation in modulating myometrial activity. Reprod. Sci. 20, 175-181. doi: 10.1177/1933719111432876

Fitzgibbon, J., Morrison, J. J., Smith, T. J., and O’Brien, M. (2009). Modulation of human uterine smooth muscle cell collagen contractility by thrombin, Y-27632, TNF alpha and indomethacin. Reprod. Biol. Endocrinol. 7, 2. doi: 10.1186/14777827-7-2

Furukawa, K., and Mattson, M. P. (1998). The transcription factor NFkappaB mediates increases in calcium currents and decreases in NMDAand AMPA/kainate-induced currents induced by tumor necrosis factor-alpha in hippocampal neurons. J. Neurochem. 70, 1876-1886. doi: 10.1046/j.14714159.1998.70051876.x

Gerhardstein, B. L., Gao, T., Bunemann, M., Puri, T. S., Adair, A., Ma, H., et al. (2000). Proteolytic processing of the C terminus of the alpha(1C) subunit of L-type calcium channels and the role of a proline-rich domain in membrane tethering of proteolytic fragments. J. Biol. Chem. 275, 8556-8563. doi: 10.1074/jbc. 275.12 .8556

Goodwin, L. O., Leeds, N. B., Guzowski, D., Hurley, I. R., Pergolizzi, R. G., and Benoff, S. (1999). Identification of structural elements of the testis-specific voltage dependent calcium channel that potentially regulate its biophysical properties. Mol. Hum. Reprod. 5, 311-322. doi: 10.1093/molehr/5.4.311

Ha, T. S., Jeong, S. Y., Cho, S. W., Jeon, H., Roh, G. S., Choi, W. S., et al. (2000). Functional characteristics of two BKCa channel variants differentially expressed in rat brain tissues. Eur. J. Biochem. 267, 910-918. doi: 10.1046/j.14321327.2000.01076.x
Hell, J. W., Westenbroek, R. E., Breeze, L. J., Wang, K. K., Chavkin, C., and Catterall, W. A. (1996). N-methyl-D-aspartate receptor-induced proteolytic conversion of postsynaptic class C L-type calcium channels in hippocampal neurons. Proc. Natl. Acad. Sci. U.S.A. 93, 3362-3367. doi: 10.1073/pnas.93.8.3362

Huang, J., Xu, L., Thomas, M., Whitaker, K., Hove-Madsen, L., and Tibbits, G. F. (2006). L-type Ca2+ channel function and expression in neonatal rabbit ventricular myocytes. Am. J. Physiol. Heart Circ. Physiol. 290, H2267-H2276. doi: 10.1152/ajpheart.01093.2005

Jurkat-Rott, K., and Lehmann-Horn, F. (2004). The impact of splice isoforms on voltage-gated calcium channel alphal subunits. J. Physiol. 554, 609-619. doi: 10.1113/jphysiol.2003.052712

Karolczak-Bayatti, M., Sweeney, M., Cheng, J., Edey, L., Robson, S. C., Ulrich, S. M., et al. (2011). Acetylation of heat shock protein 20 (Hsp20) regulates human myometrial activity. J. Biol. Chem. 286, 34346-34355. doi: 10.1074/jbc.M111.278549

Keelan, J. A., Marvin, K. W., Sato, T. A., Coleman, M., McCowan, L. M., and Mitchell, M. D. (1999). Cytokine abundance in placental tissues: evidence of inflammatory activation in gestational membranes with term and preterm parturition. Am. J. Obstet. Gynecol. 181, 1530-1536. doi: 10.1016/S00029378(99)70400-X

Kepplinger, K. J., Kahr, H., Forstner, G., Sonnleitner, M., Schindler, H., Schmidt, T., et al. (2000). A sequence in the carboxy-terminus of the alpha(1C) subunit important for targeting, conductance and open probability of Ltype $\mathrm{Ca}(2+)$ channels. FEBS Lett. 477, 161-169. doi: 10.1016/S0014-5793(00) 01791-9

Khan, I., Tabb, T., Garfield, R. E., Jones, L. R., Fomin, V. P., Samson, S. E., et al. (1993a). Expression of the internal calcium pump in pregnant rat uterus. Cell Calcium 14, 111-117.

Khan, R. N., Matharoo-Ball, B., Arulkumaran, S., and Ashford, M. L. (2001). Potassium channels in the human myometrium. Exp. Physiol. 86, 255-264. doi: 10.1113/eph8602181

Khan, R. N., Morrison, J. J., Smith, S. K., and Ashford, M. L. (1998a). Activation of large-conductance potassium channels in pregnant human myometrium by pinacidil. Am. J. Obstet. Gynecol. 178, 1027-1034.

Khan, R. N., Smith, S. K., and Ashford, M. L. (1998b). Contribution of calciumsensitive potassium channels to NS1619-induced relaxation in human pregnant myometrium. Hum. Reprod. 13, 208-213.

Khan, R. N., Smith, S. K., Morrison, J. J., and Ashford, M. L. (1993b). Properties of large-conductance $\mathrm{K}+$ channels in human myometrium during pregnancy and labour. Proc. Biol. Sci. 251, 9-15.

Khan, R. N., Smith, S. K., Morrison, J. J., and Ashford, M. L. (1997). Ca2+ dependence and pharmacology of large-conductance $\mathrm{K}+$ channels in nonlabor and labor human uterine myocytes. Am. J. Physiol. 273, C1721-C1731.

Khashan, A. S., Baker, P. N., and Kenny, L. C. (2010). Preterm birth and reduced birthweight in first and second teenage pregnancies: a register-based cohort study. BMC Pregnancy Childbirth 10:36. doi: 10.1186/1471-2393-10-36

Kim, J. M., Beyer, R., Morales, M., Chen, S., Liu, L. Q., and Duncan, R. K. (2010). Expression of BK-type calcium-activated potassium channel splice variants during chick cochlear development. J. Comp. Neurol. 518, 2554-2569. doi: 10.1002/cne.22352

Knock, G. A., and Aaronson, P. I. (1999). Calcium antagonistic properties of the cyclooxygenase-2 inhibitor nimesulide in human myometrial myocytes. Br. J. Pharmacol. 127, 1470-1478. doi: 10.1038/sj.bjp.0702685

Korovkina, V. P., Fergus, D. J., Holdiman, A. J., and England, S. K. (2001). Characterization of a novel 132-bp exon of the human maxi-channel, K. Am. J. Physiol. Cell Physiol. 281, C361-C367. Available online at: http://ajpcell. physiology.org/content/281/1/C361.full-text.pdf+html

Ledoux, J., Werner, M. E., Brayden, J. E., and Nelson, M. T. (2006). Calciumactivated potassium channels and the regulation of vascular tone. Physiology (Bethesda) 21, 69-78. doi: 10.1152/physiol.00040.2005

Leroy, M. J., Dallot, E., Czerkiewicz, I., Schmitz, T., and Breuiller-Fouch,é, M. (2007). Inflammation of choriodecidua induces tumor necrosis factor alphamediated apoptosis of human myometrial cells. Biol. Reprod. 76, 769-776. doi: 10.1095/biolreprod.106.058057

Liu, G., Shi, J., Yang, L., Cao, L., Park, S. M., Cui, J., et al. (2004). Assembly of a $\mathrm{Ca} 2+$-dependent $\mathrm{BK}$ channel signaling complex by binding to beta2 adrenergic receptor. EMBO J. 23, 2196-2205. doi: 10.1038/sj.emboj.7600228

Lu, G., Mazet, B., Sun, C., Qian, X., Johnson, C. P., Adams, M. B., et al. (1999). Inflammatory modulation of calcium-activated potassium channels in 
canine colonic circular smooth muscle cells. Gastroenterology 116, 884-892. doi: 10.1016/S0016-5085(99)70071-5

Lu, R., Alioua, A., Kumar, Y., Eghbali, M., Stefani, E., and Toro, L. (2006). MaxiK channel partners: physiological impact. J. Physiol. 570, 65-72. doi: 10.1113/jphysiol.2005.098913

Mangham, L. J., Petrou, S., Doyle, L. W., Draper, E. S., and Marlow, N. (2009). The cost of preterm birth throughout childhood in England and Wales. Pediatrics 123, e312-e327. doi: 10.1542/peds.2008-1827

Marlow, N., Wolke, D., Bracewell, M. A., and Samara, M. (2005). Neurologic and developmental disability at six years of age after extremely preterm birth. N. Engl. J. Med. 352, 9-19. doi: 10.1056/NEJMoa041367

McCartney, C. E., McClafferty, H., Huibant, J. M., Rowan, E. G., Shipston, M. J., and Rowe, I. C. (2005). A cysteine-rich motif confers hypoxia sensitivity to mammalian large conductance voltage- and Ca-activated $\mathrm{K}(\mathrm{BK})$ channel alpha-subunits. Proc. Natl. Acad. Sci. U.S.A. 102, 17870-17876. doi: 10.1073/pnas.0505270102

Mendelson, C. R. (2009). Minireview: fetal-maternal hormonal signaling in pregnancy and labor. Mol. Endocrinol. 23, 947-954. doi: 10.1210/me.2009-0016

Moynihan, A. T., Hehir, M. P., Sharkey, A. M., Robson, S. C., Europe-Finner, G. N., and Morrison, J. J. (2008). Histone deacetylase inhibitors and a functional potent inhibitory effect on human uterine contractility. Am. J. Obstet. Gynecol. 199, 167.e1-167.e7. doi: 10.1016/j.ajog.2008.01.002

Ngo, P., Ramalingam, P., Phillips, J. A., and Furuta, G. T. (2006). Collagen gel contraction assay. Methods Mol. Biol. 341, 103-109. doi: 10.1385/1-59745-113-4:103

Nijland, M. J., Ford, S. P., and Nathanielsz, P. W. (2008). Prenatal origins of adult disease. Curr. Opin. Obstet. Gynecol. 20, 132-138. doi: 10.1097/GCO. ob013e3282f76753

Oei, S. G. (2006). Calcium channel blockers for tocolysis: a review of their role and safety following reports of serious adverse events. Eur. J. Obstet. Gynecol. Reprod. Biol. 126, 137-145. doi: 10.1016/j.ejogrb.2006.03.001

Opsjln, S. L., Wathen, N. C., Tingulstad, S., Wiedswang, G., Sundan, A., Waage, A., et al. (1993). Tumor necrosis factor, interleukin-1, and interleukin-6 in normal human pregnancy. Am. J. Obstet. Gynecol. 169, 397-404. doi: 10.1016/00029378(93)90096-2

Osman, I., Young, A., Ledingham, M. A., Thomson, A. J., Jordan, F., Greer, I. A., et al. (2003). Leukocyte density and pro-inflammatory cytokine expression in human fetal membranes, decidua, cervix and myometrium before and during labour at term. Mol. Hum. Reprod. 9, 41-45. doi: 10.1093/molehr/gag001

Parkington, H. C., Tonta, M. A., Brennecke, S. P., and Coleman, H. A. (1999). Contractile activity, membrane potential, and cytoplasmic calcium in human uterine smooth muscle in the third trimester of pregnancy and during labor. Am. J. Obstet. Gynecol. 181, 1445-1451. doi: 10.1016/S0002-9378(99)70390-X

Perez, G. J., Toro, L., Erulkar, S. D., and Stefani, E. (1993). Characterization of largeconductance, calcium-activated potassium channels from human myometrium. Am. J. Obstet. Gynecol. 168, 652-660. doi: 10.1016/0002-9378(93)90513-I

Perez-Reyes, E., Wei, X. Y., Castellano, A., and Birnbaumer, L. (1990). Molecular diversity of L-type calcium channels. Evidence for alternative splicing of the transcripts of three non-allelic genes. J. Biol. Chem. 265, 20430-20436.

Phaneuf, S., Europe-Finner, G. N., Varney, M., MacKenzie, I. Z., Watson, S. P., and López Bernal, A. (1993). Oxytocin-stimulated phosphoinositide hydrolysis in human myometrial cells: involvement of pertussis toxin-sensitive and -insensitive G-proteins. J. Endocrinol. 136, 497-509. doi: 10.1677/joe.0.13 60497

Phillips, R. J., Tyson-Capper Née Pollard, A. J., Bailey, J., Robson, S. C., and Europe-Finner, G. N. (2005). Regulation of expression of the chorionic gonadotropin/luteinizing hormone receptor gene in the human myometrium: involvement of specificity protein-1 (Sp1), Sp3, Sp4, Sp-like proteins, and histone deacetylases. J. Clin. Endocrinol. Metab. 90, 3479-3490. doi: 10.1210/jc.2004-1962

Ratz, P. H., Berg, K. M., Urban, N. H., and Miner, A. S. (2005). Regulation of smooth muscle calcium sensitivity: $\mathrm{KCl}$ as a calcium-sensitizing stimulus. Am. J. Physiol. Cell Physiol. 288, C769-C783. doi: 10.1152/ajpcell.00529.2004

Romero, R., Mazor, M., Brandt, F., Sepulveda, W., Avila, C., Cotton, D. B., et al. (1992). Interleukin-1 alpha and interleukin-1 beta in preterm and term human parturition. Am. J. Reprod. Immunol. 27, 117-123. doi: 10.1111/j.16000897.1992.tb00737.x

Sadowsky, D. W., Adams, K. M., Gravett, M. G., Witkin, S. S., and Novy, M. J. (2006). Preterm labor is induced by intraamniotic infusions of interleukin1beta and tumor necrosis factor-alpha but not by interleukin- 6 or interleukin- 8 in a nonhuman primate model. Am. J. Obstet. Gynecol. 195, 1578-1589. doi: 10.1016/j.ajog.2006.06.072

Shmygol, A., Blanks, A. M., Bru-Mercier, G., Gullam, J. E., and Thornton, S. (2007). Control of uterine $\mathrm{Ca} 2+$ by membrane voltage: toward understanding the excitation-contraction coupling in human myometrium. Ann. N.Y. Acad. Sci. 1101, 97-109. doi: 10.1196/annals.1389.031

Smith, R. D., Borisova, L., Wray, S., and Burdyga, T. (2002). Characterisation of the ionic currents in freshly isolated rat ureter smooth muscle cells: evidence for species-dependent currents. Pflugers Arch. 445, 444-453. doi: 10.1007/s00424002-0941-7

Snutch, T. P., Tomlinson, W. J., Leonard, J. P., and Gilbert, M. M. (1991). Distinct calcium channels are generated by alternative splicing and are differentially expressed in the mammalian CNS. Neuron 7, 45-57. doi: 10.1016/08966273(91)90073-9

Soldatov, N. M. (1992). Molecular diversity of L-type Ca2+ channel transcripts in human fibroblasts. Proc. Natl. Acad. Sci. U.S.A. 89, 4628-4632. doi: 10.1073/pnas.89.10.4628

Soldatov, N. M. (1994). Genomic structure of human L-type Ca2+ channel. Genomics 22, 77-87. doi: 10.1006/geno.1994.1347

Soldatov, N. M., Zuhlke, R. D., Bouron, A., and Reuter, H. (1997). Molecular structures involved in L-type calcium channel inactivation. Role of the carboxylterminal region encoded by exons $40-42$ in alphalC subunit in the kinetics and $\mathrm{Ca} 2+$ dependence of inactivation. J. Biol. Chem. 272, 3560-3566. doi: 10.1074/jbc.272.6.3560

Soloff, M. S., Jeng, Y. J., Ilies, M., Soloff, S. L., Izban, M. G., Wood, T. G., et al. (2004). Immortalization and characterization of human myometrial cells from term-pregnant patients using a telomerase expression vector. Mol. Hum. Reprod. 10, 685-695. doi: 10.1093/molehr/gah086

Song, M., Zhu, N., Olcese, R., Barila, B., Toro, L., and Stefani, E. (1999). Hormonal control of protein expression and mRNA levels of the MaxiK channel alpha subunit in myometrium. FEBS Lett. 460, 427-432. doi: 10.1016/S00145793(99)01394-0

Spange, S., Wagner, T., Heinzel, T., and Krämer, O. H. (2009). Acetylation of nonhistone proteins modulates cellular signalling at multiple levels. Int. J. Biochem. Cell Biol. 41, 185-198. doi: 10.1016/j.biocel.2008.08.027

Tang, Z. Z., Liang, M. C., Lu, S., Yu, D., Yu, C. Y., Yue, D. T., et al. (2004). Transcript scanning reveals novel and extensive splice variations in human l-type voltagegated calcium channel, Cav1.2 alphal subunit. J. Biol. Chem. 279, 44335-44343. doi: 10.1074/jbc.M407023200

Tezuka, N., Ali, M., Chwalisz, K., and Garfield, R. E. (1995). Changes in transcripts encoding calcium channel subunits of rat myometrium during pregnancy. Am. J. Physiol. 269, C1008-C1017.

Tian, L., Coghill, L. S., McClafferty, H., MacDonald, S. H., Antoni, F. A., Ruth, P., et al. (2004). Distinct stoichiometry of BKCa channel tetramer phosphorylation specifies channel activation and inhibition by cAMP-dependent protein kinase. Proc. Natl. Acad. Sci. U.S.A. 101, 11897-11902. doi: 10.1073/pnas.0402590101

Tliba, O., Panettieri, R. A., Tliba, S., Walseth, T. F., and Amrani, Y. (2004). Tumor necrosis factor-alpha differentially regulates the expression of proinflammatory genes in human airway smooth muscle cells by activation of interferon-beta-dependent CD38 pathway. Mol. Pharmacol. 66, 322-329. doi: 10.1124/mol.104.001040

Toro, L., Ramos-Franco, J., and Stefani, E. (1990). GTP-dependent regulation of myometrial KCa channels incorporated into lipid bilayers. J. Gen. Physiol. 96, 373-394. doi: 10.1085/jgp.96.2.373

Torres, Y. P., Morera, F. J., Carvacho, I., and Latorre, R. (2007). A marriage of convenience: beta-subunits and voltage-dependent K+ channels. J. Biol. Chem. 282, 24485-24489. doi: 10.1074/jbc.R700022200

Tribe, R. M., Moriarty, P., and Poston, L. (2000). Calcium homeostatic pathways change with gestation in human myometrium. Biol. Reprod. 63, 748-755. doi: 10.1095/biolreprod63.3.748

Tyagi, S., Tyagi, P., Van-le, S., Yoshimura, N., Chancellor, M. B., and de Miguel, F. (2006). Qualitative and quantitative expression profile of muscarinic receptors in human urothelium and detrusor. J. Urol. 176, 1673-1678. doi: 10.1016/j.juro.2006.06.088

Wallner, M., Meera, P., Ottolia, M., Kaczorowski, G. J., Latorre, R., Garcia, M. L., et al. (1995). Characterization of and modulation by a beta-subunit of a human maxi KCa channel cloned from myometrium. Recept. Channels 3, 185-199.

Wang, W., Huang, H., Hou, D., Liu, P., Wei, H., Fu, X., et al. (2010). Mechanosensitivity of STREX-lacking BKCa channels in the colonic smooth 
muscle of the mouse. Am. J. Physiol. Gastrointest. Liver Physiol. 299, G1231-G1240. doi: 10.1152/ajpgi.00268.2010

Wang, Y. R., Li, Z. G., Fu, J. L., Wang, Z. H., Wen, Y., and Liu, P. (2011). TNF $\alpha$-induced IP3R1 expression through TNFR1/PC-PLC/PKC $\alpha$ and TNFR2 signalling pathways in human mesangial cell. Nephrol. Dial. Transplant. 26, 75-83. doi: 10.1093/ndt/gfq406

Webster, S. J., Waite, S. L., Cookson, V. J., Warren, A., Khan, R., Gandhi, S. V., et al. (2013). Regulation of GTP-binding protein (Gas) expression in human myometrial cells: a role for tumor necrosis factor in modulating Gas promoter acetylation by transcriptional complexes. J. Biol. Chem. 288, 6704-6716. doi: 10.1074/jbc.M112.440602

Wilde, D. W., and Marshall, J. M. (1988). Effects of tetraethylammonium and 4 -aminopyridine on the plateau potential of circular myometrium from the pregnant rat. Biol. Reprod. 38, 836-845. doi: 10.1095/biolreprod38.4.836

Wray, S. (2007). Insights into the uterus. Exp. Physiol. 92, 621-631. doi: 10.1113/ expphysiol.2007.038125

Xie, J., and McCobb, D. P. (1998). Control of alternative splicing of potassium channels by stress hormones. Science 280, 443-446. doi: 10.1126/science.280.5362.443

Yan, J., Olsen, J. V., Park, K. S., Li, W., Bildl, W., Schulte, U., et al. (2008). Profiling the phospho-status of the BKCa channel alpha subunit in rat brain reveals unexpected patterns and complexity. Mol. Cell. Proteomics 7, 2188-2198. doi: 10.1074/mcp.M800063-MCP200
Yang, Y., Chen, X., Margulies, K., Jeevanandam, V., Pollack, P., Bailey, B. A., et al. (2000). L-type Ca2+ channel alpha 1c subunit isoform switching in failing human ventricular myocardium. J. Mol. Cell. Cardiol. 32, 973-984. doi: $10.1006 /$ imcc. 2000.1138

Conflict of Interest Statement: The authors declare that the research was conducted in the absence of any commercial or financial relationships that could be construed as a potential conflict of interest.

Received: 22 May 2014; accepted: 20 June 2014; published online: 15 July 2014. Citation: Waite SL, Gandhi SV, Khan RN and Chapman NR (2014) The effect of trichostatin-A and tumor necrosis factor on expression of splice variants of the MaxiK and L-type channels in human myometrium. Front. Physiol. 5:261. doi: 10.3389/fphys. 2014.00261

This article was submitted to Clinical and Translational Physiology, a section of the journal Frontiers in Physiology.

Copyright (c) 2014 Waite, Gandhi, Khan and Chapman. This is an open-access article distributed under the terms of the Creative Commons Attribution License (CC BY). The use, distribution or reproduction in other forums is permitted, provided the original author(s) or licensor are credited and that the original publication in this journal is cited, in accordance with accepted academic practice. No use, distribution or reproduction is permitted which does not comply with these terms. 